\title{
The Histone Methyltransferase Inhibitor BIX01294 Inhibits HIF-1 $\alpha$ Stability and Angiogenesis
}

\author{
Su Young Oh ${ }^{1}$, Ji Yoon Seok ${ }^{1}$, Young Sun Choi', Sung Hee Lee ${ }^{2}$, Jong-Sup Bae ${ }^{1}$, and You Mie Lee ${ }^{1, *}$
}

Hypoxia-inducible factor (HIF) is a key regulator of tumor growth and angiogenesis. Recent studies have shown that, BIX01294, a G9a histone methyltransferase (HMT)-specific inhibitor, induces apoptosis and inhibits the proliferation, migration, and invasion of cancer cells. However, not many studies have investigated whether inhibition of G9a HMT can modulate HIF-1 $\alpha$ stability and angiogenesis. Here, we show that BIX01294 dose-dependently decreases levels of HIF-1 $\alpha$ in HepG2 human hepatocellular carcinoma cells. The half-life of HIF-1 $\alpha$, expression of proline hydroxylase 2 (PHD2), hydroxylated HIF-1 $\alpha$ and von Hippel-Lindau protein ( $p$ VHL) under hypoxic conditions were decreased by BIX01294. The mRNA expression and secretion of vascular endothelial growth factor (VEGF) were also significantly reduced by $\mathrm{BIX01294}$ under hypoxic conditions in HepG2 cells. BIX01294 remarkably decreased angiogenic activity induced by VEGF in vitro, ex vivo, and in vivo, as demonstrated by assays using human umbilical vein endothelial cells (HUVECs), mouse aortic rings, and chick chorioallantoic membranes (CAMs), respectively. Furthermore, BIX01294 suppressed VEGF-induced matrix metalloproteinase 2 (MMP2) activity and inhibited VEGF-induced phosphorylation of VEGF receptor 2 (VEGFR-2), focal adhesion kinase (FAK), and paxillin in HUVECs. In addition, BIX01294 inhibited VEGF-induced formation of actin cytoskeletal stress fibers. In conclusion, we demonstrated that BIX01294 inhibits HIF-1 $\alpha$ stability and VEGF-induced angiogenesis through the VEGFR-2 signaling pathway and actin cytoskeletal remodeling, indicating a promising approach for developing novel therapeutics to stop tumor progression.

\section{INTRODUCTION}

Hypoxia inducible factor-1 (HIF-1) is a crucial transcription factor that regulates the expression of target genes involved in

\footnotetext{
${ }^{1}$ Research Institute of Pharmaceutical Sciences, College of Pharmacy, National Basic Research Laboratory of Vascular Homeostasis Regulation, ${ }^{2}$ College of Nursing, Kyungpook National University, Daegu 702701, Korea

*Correspondence: lym@knu.ac.kr

Received 3 February, 2015; revised 15 March, 2015; accepted 17 March, 2015; published online 27 May, 2015
}

Keywords: angiogenesis, BIX01294, G9a HMT inhibitor, HIF-1 $\alpha$ angiogenesis, growth factor signaling, cancer cell invasion, and metastasis (Bardos and Ashcroft, 2005, Choi et al., 2014). Therefore, blocking HIF- $1 \alpha$ activity is a critical step for inhibiting tumor angiogenesis and growth (Ke and Costa, 2006; Na, 2011). HIF-1 is a heterodimer consisting of an $\alpha$ subunit and a $\beta$ subunit. HIF-1 $\alpha$ protein accumulates under hypoxic conditions, whereas HIF-1 $\beta$ is constitutively expressed. Under normoxic conditions, HIF-1 $\alpha$ is hydroxylated at oxygen-dependent degradation (ODD) domains by proline hydroxylases (PHDs). Upon hydroxylation, von Hippel-Lindau protein ( $\mathrm{pVHL}$ ), which forms the recognition component of the ubiquitin E3 ligase complex, binds to the hydroxylated ODD domain, and HIF-1 $\alpha$ gets rapidly degraded via the ubiquitin-proteasome pathway (Stroka et al., 2001; Yee et al., 2008). However, under hypoxic conditions, PHDs are inactivated and thus HIF- $1 \alpha$ is stabilized. Surprisingly, HIF-1 $\alpha$ can be stabilized under normoxic conditions by epigenetic mechanisms, i.e., silencing of key factors such as $\mathrm{pVHL}$ or PHD3 (Nguyen et al., 2013; Watson et al., 2010). Hypermethylation of $\mathrm{CpG}$ islands of $\mathrm{VHL}$ or PHD3 promoters induces gene silencing that gives rise to the activation of HIF-1 $\alpha$ in cancer cells (Hatzimichael et al., 2010; Nguyen et al., 2013). In addition, histone modification is also involved in $\mathrm{HIF}-1 \alpha$ activity. Histone deacetylase (HDAC) inhibitors, such as trichostatin $A$ (TSA) and suberoylanilide hydroxamic acid (SAHA), suppress angiogenesis by the angiogenic stimuli generated by HIF-1 $\alpha$ (Ellis et al., 2009; Kuljaca et al., 2007; Xu et al., 2007).

Induction of the vascular endothelial growth factor (VEGF) by HIF-1 plays a key role in angiogenesis and represents an important step in tumor growth (Forsythe et al., 1996; Kiselyov et al., 2007). VEGF and VEGF receptor-2 (VEGFR-2) have been implicated in a major endothelial cell-specific factor signaling pathway required for pathological angiogenesis, including tumor neovascularization (Mamahon, 2000). Another central mediator of VEGF signaling in endothelial cells is the focal adhesion kinase (FAK) pathway that plays an important role in actin cytoskeleton organization and cell motility (Abedi and Zachary, 1997). EZH2, an HMT decreases vasohibin1, an antiangiogenic factor by the promoter histone methylation. It suggests that $\mathrm{EZH} 2$ increases angiogenesis indirectly by the inhibition of a target gene, vasohibin1 (Lu et al., 2010).

The G9a histone methyltransferase (HMT) methylates lysine 9 (Lys9) of histone H3. It has been reported to promote both cell proliferation and metastasis in various types of cancers (Chen et al., 2010). Therefore, it is suggested that inhibition of G9a HMT could be used as a therapeutic approach in cancer treatment. BIX01294, a G9a HMT inhibitor, decreases cell mobility, proliferation, invasion, and migration in neuroblastoma ( $\mathrm{Lu}$ 
et al., 2013). BIX01294 suppresses the growth of cancer cells and induces cell death, suggesting that BIX01294 is an effective antitumor drug (Kim et al., 2013; Varier and Timmers, 2011). However, the effects of BIX01294 on HIF-1 $\alpha$ stability and tumor angiogenesis remain unclear. Here, we demonstrate that BIX01294 decreases HIF-1 $\alpha$ stability under hypoxic conditions in HepG2 cells. In addition, BIX01294 suppresses VEGF/ VEGFR-2 signaling, especially FAK and paxillin, in human umbilical vein endothelial cells (HUVECs).

\section{MATERIALS AND METHODS}

\section{Cell culture}

HepG2 cells were obtained from the Korean Cell Line Bank (Korea) and maintained in the DMEM containing 10\% FBS, $1 \%$ penicillin/streptomycin $(10,000 \mathrm{U} / \mathrm{ml})$. HUVECs were obtained from InnoPharmaScreen (Korea), cultured on 1\% gelatincoated dishes, and maintained in M199 medium supplemented with $20 \% \mathrm{FBS}, 2 \mathrm{ng} / \mathrm{ml} \mathrm{bFGF,} 2 \mathrm{U} / \mathrm{ml}$ heparin, and $1 \%$ penicillin/streptomycin $(10,000 \mathrm{U} / \mathrm{ml})$.

\section{MTT assay}

Cells were treated with different concentrations of BIX01294 (Enzo, USA). After 24 h, 3-(4,5-dimethylthiazol-2-yl)-2,5-diphenyl tetrazolium bromide (MTT; Sigma) reagent was added to each well. Precipitated formazan was dissolved in DMSO, and the optical density was measured at $540 \mathrm{~nm}$.

\section{Western blotting}

Cells were harvested with a lysis buffer (iNtRon Biotech, Korea). Equal amounts of protein were subjected to SDS-PAGE and proteins were transferred to nitrocellulose membranes (Whatman, England). The membranes were blocked for $1 \mathrm{~h}$ with $5 \%$ low-fat milk-powder TBST (50 mM Tris- $\mathrm{HCl}, \mathrm{pH} 7.5,150 \mathrm{mM} \mathrm{NaCl}$, $0.1 \%$ Tween-20) and then incubated with antibodies against HIF-1 $\alpha$, obtained from BD Bioscience (Germany); p-VEGFR-2, p-FAK, and FAK, obtained from Cell Signaling (USA); p-paxillin and paxillin, obtained from Abcam (UK); and $\beta$-actin, obtained from Santa Cruz (USA). This was followed by incubation with horseradish peroxidase-conjugated secondary antibody and ECL detection (BioRad, USA) according to the manufacturer's instructions.

\section{Reverse transcription PCR}

Total RNA was extracted using TRIzoL reagent (Invitrogen, USA) and cDNA synthesis from RNA was performed using the first strand cDNA synthesis kit (Promega, USA) according to the manufacturer's instructions. Amplification of cDNA by PCR was performed using an automated thermal cycler (BioRad, USA). Oligonucleotide primers for PCR were designed as follows: $\beta$-actin, 5 '-gactacctcatgaagatc- $3^{\prime}$ and 5 '-gatccacatctgctggaa-3'; HIF-1 $\alpha, 5^{\prime}$-cagaagatacaagtagcctc-3' and $5^{\prime}$-ctgctggaatactgtaactg-3'; VEGF, 5'-gggcagagctgagtgttagc-3' and 5'tctcccagatcggtgacagt- 3 '.

\section{Enzyme-linked immunosorbent assay (ELISA)}

HepG2 cells were plated in 60-mm dishes and cultured to approximately $80-90 \%$ confluence. The level of VEGF protein secreted by the cells into the medium was determined with a VEGF ELISA kit (R\&D Systems, USA) according to the manufacturer's instructions. gelatin-coated 96-well plates. After $24 \mathrm{~h}$, cells were starved with a low serum medium (0.5\% FBS in M199) for $16 \mathrm{~h}$, and the medium was replaced by a low serum media containing VEGF in the presence or absence of BIX01294 for $24 \mathrm{~h}$. The cell proliferation rate was measured by $\mathrm{BrdU}$ incorporation using the Cell Proliferation ELISA, BrdU (colorimetric) kit (Roche, Germany) according to the manufacturer's instructions.

\section{Tube formation assay}

HUVECs were seeded in 96-well plates coated with Matrigel (BD Bioscience, Germany) and then incubated for $6 \mathrm{~h}$ with or without BIX01294 in the M199 media containing 1\% FBS and $20 \mathrm{ng} / \mathrm{ml}$ VEGF. Morphological changes in cells were photographed under a microscope to assess the formation of capillary-like tubes.

\section{Migration assay}

The migration assay was performed using the Transwell system (6.5 mm diameter, $8 \mu \mathrm{m}$ pore size, Corning Costar) HUVECs were starved in serum-free M199 media for $24 \mathrm{~h}$ and seeded $\left(5 \times 10^{4}\right.$ cells) in the upper chamber with or without BIX01294. The M199 media with or without BIX01294 and 20 $\mathrm{ng} / \mathrm{ml}$ VEGF were added to the lower chamber. After the cells were incubated for $24 \mathrm{~h}$, they were fixed with methanol and the filter was stained with hematoxylin and eosin.

\section{Chorioallantoic membrane (CAM) assay}

The chick egg-shell membrane and egg albumin were removed in 3.5 days after fertilization. At day 6 , retinoic acid (RA) and BIX01294 were loaded onto the CAMs of the chick embryos. After 2 days, $10 \%$ fat emulsion was injected into the chorioallantois and the CAMs were observed under a microscope. Ten eggs per group were used in each experiment, and three independent experiments were performed in total. Percentages of eggs positive for new blood vessel formation among all eggs tested were calculated.

\section{Gelatin zymography}

The conditioned medium was obtained from the cultured cells and electrophoresed under non-reducing conditions in $10 \%$ SDS-PAGE gels containing $1 \mathrm{mg} / \mathrm{ml}$ gelatin. After overnight incubation in the zymography reaction buffer $(50 \mathrm{mM}$ Tris $\mathrm{pH}$ $7.8,10 \mathrm{mM} \mathrm{CaCl}_{2}, 150 \mathrm{mM} \mathrm{NaCl}$, and $2 \mu \mathrm{M} \mathrm{ZnCl}_{2}$ ) at $37^{\circ} \mathrm{C}$, gels were stained with the Coomassie blue solution containing $10 \%$ acetic acid and $20 \%$ methanol, followed by destaining with a $10 \%$ acetic acid solution. A clear zone against the blue-stained gelatin background indicated areas of gelatinase activity.

\section{Immunofluorescence}

For cytoskeletal staining, cells were fixed with $4 \%$ formaldehyde in PBS $(\mathrm{v} / \mathrm{v})$ for $5 \mathrm{~min}$ at room temperature. After the cells were fixed, they were blocked in blocking buffer $0.5 \%$ BSA in PBS) and then incubated with Alexa Fluor ${ }^{\circledR} 488$ phalloidin (Invitrogen, USA) for $30 \mathrm{~min}$. Cells were counterstained with 4,6diamidino-2-phenylindole dihydrochloride (DAPI) and visualized by confocal microscopy (TCS-SP5, Leica Microsystems).

Chick embryo aortic ring assay

The chick thoracic aorta was obtained from 13-d-old embryos. Excess perivascular tissue was removed and the aorta was cut into transverse sections (1-2 mm). Each ring was embedded in Matrigel in 48-well plates, covered with Matrigel, and incubated with VEGF alone or a combination of VEGF and BIX01294 The plates were incubated at $37^{\circ} \mathrm{C}$ in $5 \% \mathrm{CO}_{2}$ for $24-36 \mathrm{~h}$, and 
A

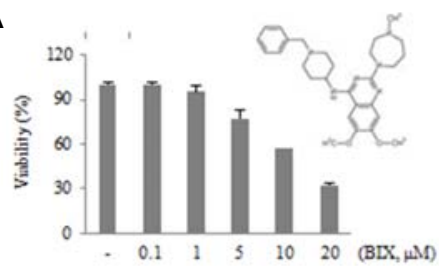

B
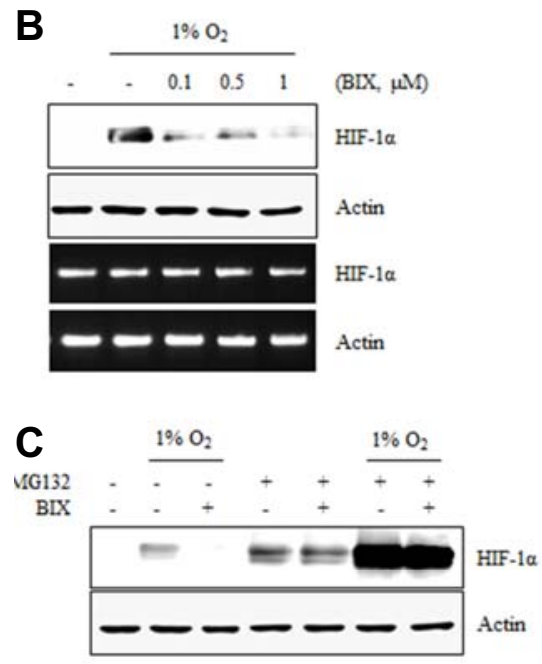

D

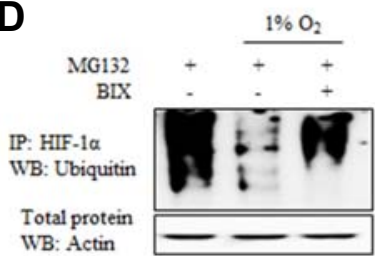

E

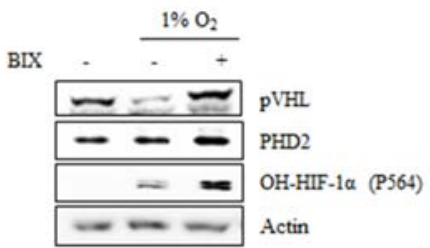

$\mathbf{F}$

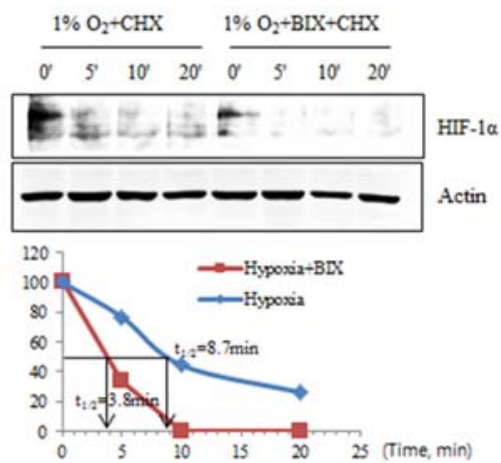

G

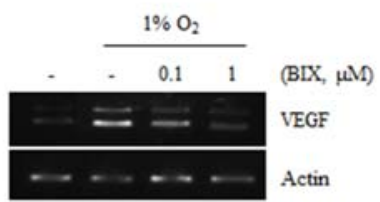

H

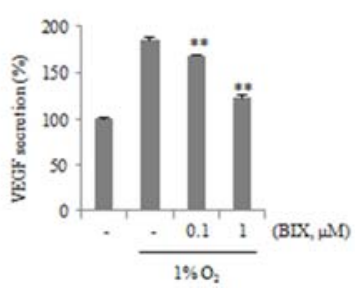

Fig. 1. BIX01294 inhibits hypoxia-induced HIF- $1 \alpha$ stability and VEGF expression. (A) HepG2 cells were treated with different concentrations of BIX01294. After $24 \mathrm{~h}$, cell viability was measured by the MTT assay. (B) HepG2 cells were exposed to hypoxic conditions for $24 \mathrm{~h}$ in the presence of 0.1-1 $\mu \mathrm{M}$ BIX01294. Expression of HIF$1 \alpha$ was measured by Western blot and PCR. (C) BIX01294 (1 $\mu$ M)-treated HepG2 cells were exposed to hypoxic conditions for $24 \mathrm{~h}$ in the presence or absence of MG132 (100 $\mu \mathrm{M}, 6 \mathrm{~h})$ and cell lysates were analyzed by Western blot. (D) HepG2 cells were exposed to hypoxic conditions in the presence of BIX01294 (1 $\mu \mathrm{M})$ and MG132 $(100 \mu \mathrm{M})$ for $6 \mathrm{~h}$. The cell lysates were imunoprecipitated with an anti-HIF-1 $\alpha$ antibody. Immunoprecipitates were subsequently immunoblotted with an anti-ubiquitin antibody. (E) HepG2 cells were exposed to hypoxic conditions for 24 $\mathrm{h}$ in the presence or absence of $1 \mu \mathrm{M}$ BIX01294. Expression of PHD2, pVHL and hydroxylated-HIF-1 $\alpha$ was measured by Western blot. (F) HepG2 cells were exposed to hypoxic conditions for $24 \mathrm{~h}$ in the presence of $\mathrm{BIX01294}$ and then treated with cycloheximide $(\mathrm{CHX}, 100 \mu \mathrm{M})$ for the durations indicated. HIF-1 $\alpha$ protein levels were determined by Western blot. (G) HepG2 cells were exposed to hypoxic conditions in the presence or absence of $\mathrm{BIX01294}$ for $24 \mathrm{~h}$ and then VEGF expression levels were determined by PCR. $(H)$ ELISA was used to measure the levels of secreted VEGF protein in conditioned media. Beta-actin was used for an internal control. ${ }^{* *} p<0.01$ versus hypoxic control. sprouting microvessels branches from each aortic ring were photographed and the number were counted.

\section{Matrigel plug assay}

BIX01294 $(1.5 \mu \mathrm{g} / \mathrm{ml})$ was mixed with the liquid Matrigel solution containing heparin $(25 \mu \mathrm{g} / \mathrm{ml})$ and VEGF $(1.5 \mu \mathrm{g} / \mathrm{ml})$ and then injected into the subcutaneous tissue of the abdominal wall. After 7 days, the Matrigel plug was removed and the resulting neovessel formation was analyzed by microscopy. Blood vessel density was quantified by Image $\mathrm{J}$ software and represented as a bar diagram.

\section{RESULTS}

BIX01294 inhibits HIF-1 $\alpha$ expression in HepG2 cells $\mathrm{BIX01294}$ was not toxic to the HepG2 cells at a concentration range of $0.1-1 \mu \mathrm{M}$, but it caused cell death at higher concentrations 5-20 $\mu \mathrm{M}$, as described previously (Lu et al., 2013) (Fig. 1A). Therefore, we used concentrations below $1 \mu \mathrm{M}$ to study the action of BIX01294 on HIF-1 $\alpha$ stability and angiogenesis. To determine the effects of BIX01294 on HIF- $1 \alpha$ activation, HepG2 cells were exposed to hypoxic conditions for $24 \mathrm{~h}$ in the presence or absence of BIX01294 (0.1-1 $\mu \mathrm{M})$. BIX01294 dosedependently suppressed HIF-1 $\alpha$ expression levels under hypoxic conditions in HepG2 cells, but did not change HIF-1 $\alpha$ mRNA levels (Fig. 1B). Treatment with BIX01294 did not alter the expression of HIF-1 $\alpha$ in the presence of MG132, a proteasome inhibitor (Fig. 1C), suggesting that BIX01294 inhibits HIF-1 $\alpha$ stability by proteasomal degradation, but not by protein synthesis inhibition. Immunoprecipitation of HIF-1 $\alpha$ followed by western blot analysis with the ubiquitin antibody showed that BIX01294 increased ubiquitination of HIF-1 $\alpha$ (Fig. 1D). In addition, BIX01294 increased the expression of PHD2, pVHL and hydroxylation of HIF-1 $\alpha$ (Fig. 1E). BIX01294 decreased the half-life of HIF-1 $\alpha$ compared to the control (Fig. 1F). These results suggest that $\mathrm{BIX01294}$ reduces the stability of HIF-1 $\alpha$ by activating HIF-1 $\alpha$ degradation through increased hydroxylation mediated by PHD2 and PVHL.

\section{BIX01294 inhibits hypoxia-induced VEGF expression in HepG2 cells}

Since VEGF is a potent mediator of hypoxia-induced angiogenesis and is regulated by HIF-1 $\alpha$ (Carroll and Ashcroft, 2008), we sought to determine whether BIX01294 could affect hypox- 
A

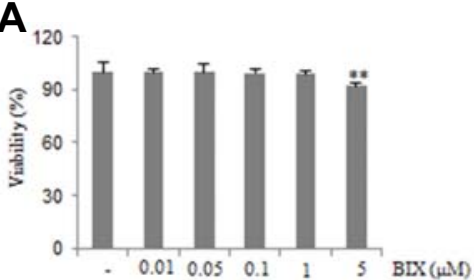

C
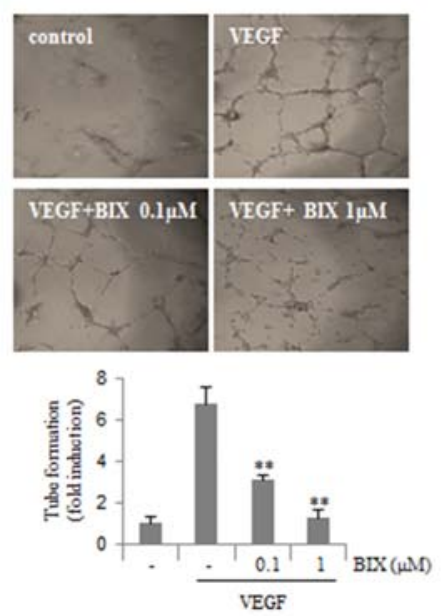

B

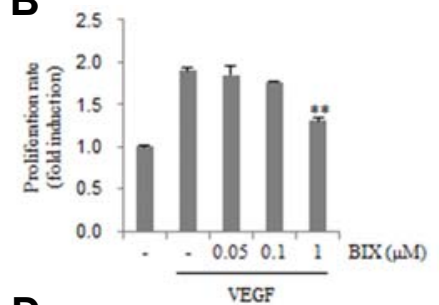

D
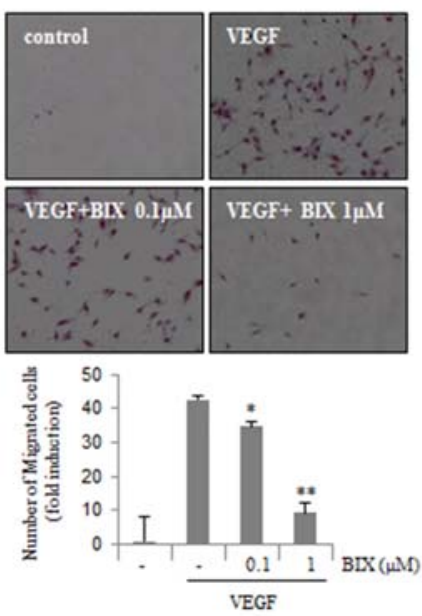

Fig. 2. BIX01294 inhibits angiogenesis. (A) HUVECs were exposed to different concentrations of BIX01294. After $24 \mathrm{~h}$, cell viability was measured by the MTT assay. (B) A BrdU-based cell proliferation assay was performed $24 \mathrm{~h}$ after treatment with BIX01294. (C) HUVECs were loaded onto the Matrigel-coated plates and treated with VEGF $(20 \mathrm{ng} / \mathrm{ml})$ in the presence or absence of BIX01294. Microphotographs were taken after 8 h. ${ }^{* *} p<0.01$ versus VEGF control. (D) HUVECs were plated onto 24-well Transwell membranes coated with collagen and treated with BIX01294 or vehicle in the presence of VEGF $(20 \mathrm{ng} / \mathrm{ml})$ for 24 h. Cells that migrated into the bottom part of the membrane were stained with hematoxylin and eosin. ${ }^{*} p<0.05$; ${ }^{* *} p<0.01$ versus VEGF control. (E) Zymograms of serum-free conditioned medium of HUVECs treated with BIX01294 $(1 \mu \mathrm{M})$ for $24 \mathrm{~h}$. A clear zone of gelatin digestion indicates the presence of MMP-2.

$\mathbf{E}$

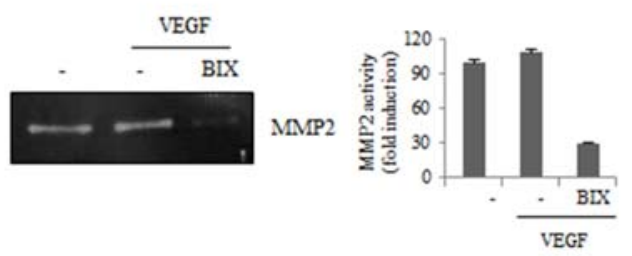

ia-induced VEGF expression and secretion. Hypoxia-induced VEGF mRNA expression (Fig. 1G) and VEGF secretion (Fig. 1H) were significantly decreased by BIX01294 in HepG2 cells. These results indicated that BIX01294 inhibited HIF-1 $\alpha$-mediated VEGF expression in HepG2 cells under hypoxic conditions.

\section{BIX01294 inhibits angiogenesis in HUVECs}

We investigated whether VEGF-induced angiogenesis could be modulated by BIX01294 in HUVECs. BIX01294 exhibited no cytotoxicity in HUVECs at concentrations of up to $1 \mu \mathrm{M}$, although increased cytotoxicity has been observed at a dose of 5 $\mu \mathrm{M}$ (Fig. 2A). Therefore, we used BIX01294 at concentrations below $1 \mu \mathrm{M}$ in this assay. BIX01294 significantly inhibited proliferation induced by VEGF in a dose-dependent manner (Fig. 2B). The tube formation of HUVECs induced by VEGF on Matrigel was dramatically disrupted by BIX01294 (Fig. 2C). When cells were treated with VEGF alone, HUVECs efficiently migrated to the bottom of the membrane (pore size, $8 \mu \mathrm{m}$ ). However, the addition of BIX01294 inhibited VEGF-induced migration of HUVECs (Fig. 2D). These results indicated that BIX01294 blocked the migration and tube-formation ability of endothelial cells induced by VEGF.

Matrix metalloproteinases (MMPs) comprise a family of endopeptidases that degrade extracellular matrix structural proteins and regulate endothelial cell migration (Karagiannis and Popel, 2006; Wang et al., 2006). Because endothelial cells activated by VEGF secrete MMPs during angiogenesis through degradation of extracellular matrix proteins (Rundhaug, 2003), we next determined whether BIX01294 can inhibit VEGFinduced secretion of MMP-2 in a gelatin zymography assay. As shown in Fig. 2E, VEGF-induced MMP-2 activity was significantly decreased in BIX01294-treated HUVECs. These results indicated that BIX01294 inhibited VEGF-induced angiogenic responses in vitro.

\section{BIX01294 inhibits ex vivo and in vivo angiogenesis}

To confirm the inhibitory effect of BIX01294 on angiogenesis induced by VEGF, we used the ex vivo chick embryo aortic ring angiogenic assay. The aortic rings were isolated from 14-d-old chick embryos and cultured in VEGF-containing serum free media in the presence or absence of BIX01294. VEGF-induced endothelial cell outgrowth from aortic ring was reduced by BIX01294 (Fig. 3A). Next, we performed the chick chorioallantoic membrane (CAM) assay. When 5-d-old chick CAMs were treated with retinoic acid (RA), a strong angiogenic inhibitor, little vascular sprouting was found during CAM development (Fig. 3B). Similar to the case with RA-treated CAMs, new sprouting of microvessels from large vessels was significantly 


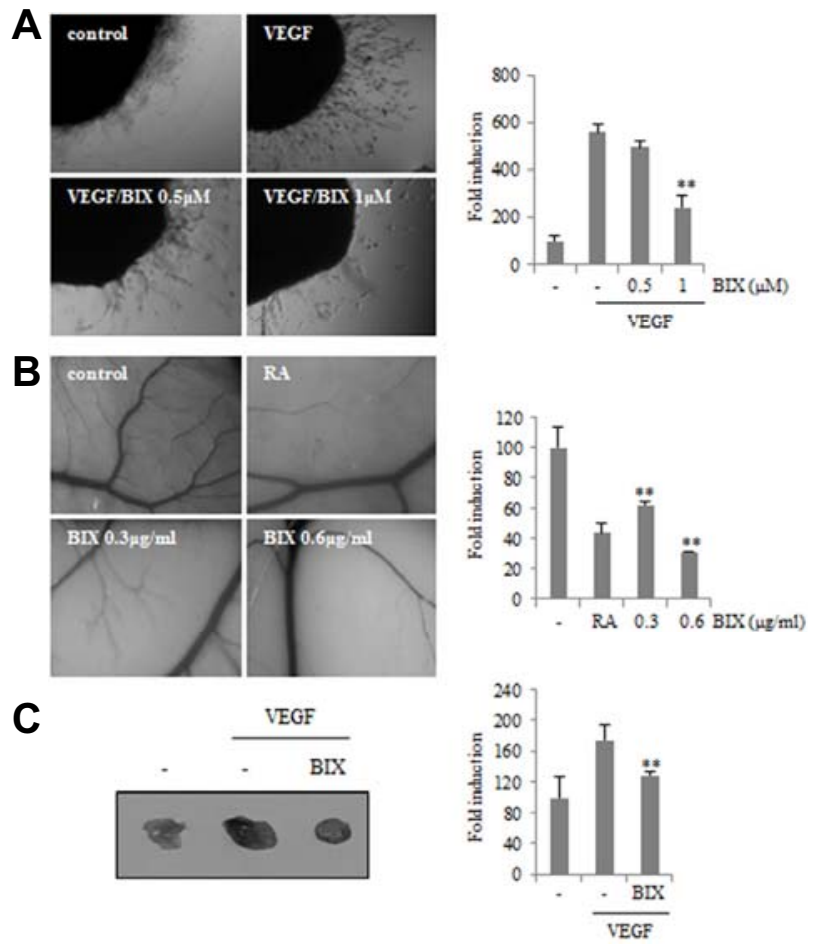

Fig. 3. Effect of BIX01294 on the growth of new blood vessels. (A) Chick embryo aortas in Matrigel were treated with VEGF $(20 \mathrm{ng} / \mathrm{ml})$ in the absence or presence of BIX01294 for $24 \mathrm{~h}$. Representative aortic rings were photographed. Three independent experiments were performed in triplicate. ${ }^{* *} p<0.01$ versus VEGF control. (B) Thermonox cover slips with BIX01294 or retinoic acid (RA, $1 \mu \mathrm{g}$ ) were loaded onto CAMs. After 3 days, a fat emulsion was injected under CAMs for better visualization of the vessels. The images shown are representative photographs of the chick CAM assay. Percentages of eggs positive for new vessel formation among all eggs tested were calculated. ${ }^{* *} p<0.01$ versus control. (C) Mice were injected with Matrigel containing VEGF $(2.5 \mu \mathrm{g} / \mathrm{ml})$ with or without BIX01294 $(0.6 \mu \mathrm{g} / \mathrm{ml})$ into the flank of C57BL6/J mice. After 7 days, the plugs were removed and blood vessels density was quantified by Image $\mathrm{J}$ software and represented as a bar diagram. ${ }^{* *} p<0.01$ versus VEGF control.

inhibited in BIX01294-treated embryos compared to that in control embryos. To determine if BIX01294 can suppress VEGFinduced angiogenesis in vivo, mice were injected with $200 \mu \mathrm{l}$ of VEGF containing Matrigel with or without BIX01294. VEGF induced endothelial cells from the neighboring blood vessels to develop functional neo-vessels into the Matrigel plug. BIX01294 treatment reduced the extent of VEGF-induced microvessel formation within the Matrigel plug (Fig. $3 \mathrm{C}$ ). These results demonstrated that BIX01294 potently blocked in vivo neovascularization.

BIX01294 inhibits the phosphorylation of VEGFR-2 and FAK

Because BIX01294 inhibited the VEGF-induced tube formation, proliferation, and migration of HUVECs, we investigated whether BIX01294 could inhibit VEGF-induced VEGFR-2 activation. Activation of VEGFR-2 by phosphorylation increased after VEGF treatment for 15 min, but treatment with BIX01294 significantly inhibited VEGFR-2 phosphorylation (Fig. 4A).
A

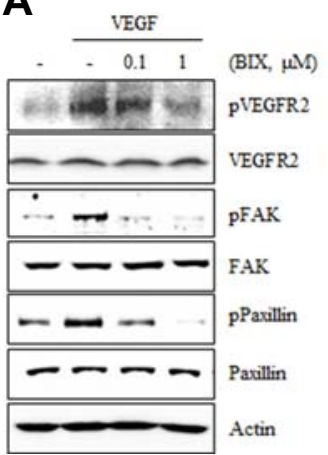

B

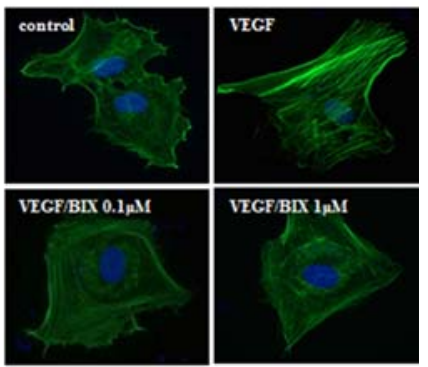

Fig. 4. BIX01294 suppresses VEGFR-2 and FAK-mediated signaling pathways. (A) HUVECs were treated with various concentrations of BIX01294 and stimulated with VEGF $(20 \mathrm{ng} / \mathrm{ml})$ for $15 \mathrm{~min}$. Activation of VEGFR-2, FAK, and paxillin was determined by Western blot. (B) HUVECs were stimulated with VEGF $(20 \mathrm{ng} / \mathrm{ml})$ in the presence or absence of BIX01294, and then filamentous actin was visualized by immunofluorescent staining using Alexa Fluor 488conjugated phalloidin.

FAK is known to regulate endothelial cell survival, proliferation, migration, and tumor-associated angiogenesis (Abedi and Zachary, 1997; Cabrita et al., 2011; Tavora et al., 2010; Tong et al., 2014). As FAK can be rapidly activated by VEGFR-2 signaling, we investigated potential effects of BIX01294 on FAK activation. VEGF-induced FAK phosphorylation was significantly decreased by pretreatment with BIX01294 in HUVECs (Fig. 4A).

Since paxillin is a downstream molecule of FAK that controls endothelial cell migration and tumor angiogenesis (German et al., 2014; Schaller, 2001), we investigated whether phosphorylation of paxillin was affected by BIX01294 (Fig. 4A). Because FAK and paxillin are involved in cytoskeletal dynamics, we investigated VEGF-induced cytoskeletal reorganization by phalloidin staining. We observed that VEGF caused marked actin cytoskeletal reorganization characterized by formation of microfilament stress fibers in HUVECs, whereas pretreatment with BIX01294 prevented this process (Fig. 4B). These results suggested that BIX01294 inhibited VEGF-induced actin reorganization into stress fibers through FAK and paxillin inactivation.

\section{DISCUSSION}

Epigenetic changes in DNA methylation and histone modification are frequently implicated in driving tumorigenesis and angiogenesis. Drugs that prevent such epigenetic changes have been increasingly proposed as potential therapeutics for cancer, especially in the case of HIF-1 $\alpha$. The DNA methyltransferase (DNMT) inhibitor 5-aza-2'-deoxycitidine (5-aza-dC) increases HIF- $1 \alpha$ mRNA expression levels and HIF- $1 \alpha$ binding to the promoter of a target gene, such as stanniocalcin-2 (Law et al., 2008). Treatment with TSA, an HDAC inhibitor, also increases expression of pVHL, an E3 ubiquitin ligase for HIF-1 $\alpha$ (Nguyen et $\mathrm{al}, 2013$ ). The role of epigenetic modifications in HIF-1 $\alpha$ activation has not been studied in detail. There have been conflicting reports about the enhancement and suppression of HIF-1 $\alpha$ activation by pontin and reptin, respectively (targets of G9a/GLP methyltransferase (Lee et al, 2010; 2011). In this study, we sought to elucidate whether inhibition of the G9a 
HMT can modulate activation of HIF- $1 \alpha$. We observed that treatment with BIX01294, a G9a HMT inhibitor, increased PHD2 and pVHL expression and diminished HIF-1 $\alpha$ stability by proteasomal degradation in hypoxia.

It has been reported that, at concentrations higher than $1 \mu \mathrm{M}$, BIX01294 induces cell cycle arrest in the G1 phase, apoptosis, and intracellular ROS accumulation in breast and colon cancer cells (Lu et al., 2013; Schaller, 2001). In our experiments, the expression of p21 and levels of ROS generation were not increased by $\sim 1 \mu \mathrm{M}$ BIX01294. In addition, no increase in the expression of LC3, an autophagosome marker, was observed in HepG2 cells. However, at a relatively high dose of $10 \mu \mathrm{M}$, BIX01294 induced p21 protein expression in HepG2 cells (data not shown), as has been documented previously (Lu et al., 2013). In fact, in our experiments a lower concentration of BIX01294 $(\sim 1 \mu \mathrm{M})$ increased ubiquitination and subsequent degradation of HIF-1 $\alpha$ (Fig. 1), which may explain the inhibition of VEGF secretion (Fig. 1). Moreover, BIX01294 suppressed VEGF-induced tube formation, migration of HUVECs, and in vivo neovascularization (Figs. 2 and 3). In addition, BIX01294 decreased VEGF-induced phosphorylation of VEGFR-2, FAK and paxillin, as well as the formation of stress fibers (Fig. 4). These results suggest that BIX01294 can be effective against angiogenesis at lower concentrations $(\sim 1 \mu \mathrm{M})$ and antiangiogenic properties of BIX01294 are could be mediated by the disruption of VEGFR-2/FAK/paxillin-mediated angiogenesis signaling.

VEGF receptor signaling induces EZH2 expression and then $\mathrm{EZH} 2$ promotes inhibits expression of target genes including vasohibin1, an anti-angiogenic molecules (Lu et al., 2010). Therefore, it is suggested that EZH2 inhibits anti-angiogenic molecules by the histone methylation. Because BIX01294 inhibits HMT activity of G9a by specific binding to its SET domain (Chang et al., 2009), inhibition of VEGF-induced angiogenesis by BIX01294 may be an indirect effect, i.e. some VEGF signaling molecules or other angiogenic molecules are upregulated by G9a inhibition. Here, we did not verify precise action mechanism of BIX01294 in HIF-1 $\alpha$ stability and VEGF-induced angiogenesis. However, the results that BIX01294 increased pVHL level and hydroxylation of HIF-1 $\alpha$ may explain one of the mechanisms of anti-angiogenic effect in cancer cells.

Combination epigenetic therapy for the treatment of tumors has recently become a primary focus of clinical development efforts (Sigalotti et al., 2014). Our data suggest that, when used at a relatively low dose, BIX01294 has considerable potential as a component of combined epigenetic therapies that would allow overcoming its dose-limiting toxicity. Including BIX01294 in combination therapy would also help to optimize the use of other epigenetic drugs and antitumor agents by lowering their potentially toxic doses. The present data warrant further investigation of the molecular mechanisms involved in the regulation of HIF-1 $\alpha$ stability and VEGF signaling by BIX01294.

\section{ACKNOWLEDGMENTS}

This work was supported by the NRF grant funded by the Korean government (MSIP) (2012R1A4A1028835 and 2013R1 A2A2A01068868).

\section{REFERENCES}

Abedi, H., and Zachary, I. (1997). Vascular endothelial growth factor stimulates tyrosine phosphorylation and recruitment to new focal adhesions of focal adhesion kinase and paxillin in endothelial cells. J. Bio. Chem. 272, 15442-15451.

Bardos, J.I. and Ashcroft, J. (2005). Negative and positive regulation of HIF-1: a complex network. Biochimica et Biophysica Acta $1755,107-120$.
Cabrita, M.A., Jones, L.M., Quizi, J.L., Sabourin, L.A., Makay, B.C., and Addison, C.L. (2011). Focal adhesion kinase inhibitors are potent anti-angiogenic agents. Mol. Oncol. 5, 517-526.

Carroll, V.A., and Ashcroft, M. (2008). Role of hypoxia-inducible factor (HIF)- $1 \alpha$ versus HIF-2 $\alpha$ in the regulation of HIF target genes in response to hypoxia, insulin-like growth factor-I, or loss of von hippel-lindau function: implications for targeting the HIF pathway. Cancer Res. 15, 6264-6270.

Chang, Y., Zhang, X., Horton, J.R., Upadhyay, A.K., Spannhoff, A., Liu, J., Snyder, J.P., Bedford, M.T., and Cheng, X. (2009). Structural basis for G9a-like protein lysine methyltransferase inhibition by BIX-01294. Nat. Struct. Mol. Biol. 16. 312-317.

Chen, M.W., Hua, K.T., Kao, H.J., Chi, C.C., Wei, L.H., Johansson, G., Shiah, S.G., Chen, P.S., Jeon, Y.M, Cheng, T.Y., et al. (2010). H3K9 histone methyltransferase G9a promotes lung cancer invasion and metastasis by silencing the cell adhesion molecule Ep-CAM. Cancer Res. 70, 7830-7840.

Choi, J.H., Nguyen, M.P., Lee, D., Oh, G.T., and Lee, Y.M. (2014). Hypoxia-induced endothelial progenitor cell function is blunted in angiotensinogen knockout mice. Mol. Cells 37, 487-496.

Ellis, L., Hammers, H., and Pili, R. (2009). Targeting tumor angiogenesis with histone deacetylase inhibitors. Cancer Lett. 280, 145- 153.

Forsythe, J.A., Jiang, B.H., Iyer, N.V., Agani, F., Leung, S.W., Koos, R.D., and Semenza, G.L. (1996). Activation of vascular endothelial growth factor gene transcription by hypoxia-inducible factor 1. Mol. Cell. Biol. 16, 4604-4613.

German, A.E., Mammoto, T., Jiang, E., Ingber, D.E., and Mammoto, A. (2014). Paxillin controls endothelial cell migration and tumor angiogenesis by altering neuropilin 2 expression. J. Cell Sci. 127, 1672-1683.

Hatzimichael, E., Dasoula, A., Shah, R., Syed, N., Papoudou-Bai, A., Coley, H.M., Dranitasris, G., Bourantas, K.L., Stebbing, J., and Crook, T. (2010). The prolyl-hydroxylase EGLN3 and not EGLN1 is inactivated by methylation in plasma cell neoplasia. Eur. J. Haematol. 84, 47-51.

Karagiannis, E.D., and Popel, A.S. (2006). Distinct modes of collagen type I proteolysis by matrix metalloproteinase (MMP) 2 and membrane type I MMP during the migration of a tip endothelial cell: insights from a computational model. J. Theor. Biol. 238 124-145.

Ke, Q., and Costa, M. (2006). Hypoxia-inducible factor-1 (HIF-1). Mol. Pharm. 70, 1469-1480.

Kim, Y., Kim, Y.S., Kim, D.E., Lee, J.S., Song, J.H., Kim, H.G., Cho, D.H., Jeong, S.Y., Jin, D.H., Jang, S.J. et al. (2013). BIX-01294 induces autophagy-associated cell death via EHMT2/G9a dysfunction and intracellular reactive oxygen species production. Autophagy 9, 2126-2139.

Kiselyov, A., Balakin, K.V., and Tkachenko, S.E. (2007). VEGF/VEGFR signaling as a target for inhibiting angiogenesis. Pepert. Pion. Investig. Drugs 16, 83-107.

Kuljaca, S., Liu, T., Tee, A.E.L., Haber, M. Norris, Dwarte, G.M., and Marshall, M.D.T. (2007). Enhancing the anti-angiogenic action of histone deacetylase inhibitors. Mol. Cancer 6, 1-11.

Law, A.Y., Lai, K.P., Ip, C.K., Wong, A.S., Wagner, G.F., and Wong, C.K. (2008). Epigenetic and HIF-1 regulation of stanniocalcin-2 expression in human cancer cells. Exp. Cell Res. 314, 18231830.

Lee, J.S., Kim, Y., Kim, I.S., Kim, B., Choi, H.J., Lee, J.M., Shin, H.J., Kim, J.H., Kim, J.Y., Seo, S.B., et al. (2010). Negative regulation of hypoxic responses via induced reptin methylation. Mol. Cell 39, 71-85.

Lee, J.S., Kim, Y., Bhin, J., Shin, H.J., Nam, H.J., Lee, S.H., Yoon, J.B., Binda, O., Gozani, O., Hwang, D., et al. (2011). Hypoxiainduced methylation of a pontin chromatin remodeling factor. Proc. Natl. Acad. Sci. USA 108, 13510-13515.

Lu, C., Han, H.D., Mangala, L.S., Ali-Fehmi, R., Newton, C.S Ozbun, L., Armaiz-Pena, G.N., Hu, W., Stone, R.L., Munkarah, A., et al. (2010). Regulation of tumor angiogenesis by EZH2. Cancer Cell 18, 185-197.

Lu, Z., Tian, Y. Salwen, H.R., Chlenski, A., Godley, L.A., Raj, J.U., and Yang, Q. (2013). Histone-lysine methyltransferase EHMT2 is involved in proliferation, apoptosis, cell invasion, and DNA methylation of human neuroblastoma cells. Anti-Cancer Drugs 24, 484-493

Ma, N. (2011). HIF-1 is the commander of gateways to cancer. 
Nagy J. Cancer Sci. Ther. 3, 35-40.

Mamahon, G. (2000). VEGF receptor signaling in tumor angiogenesis. Oncologist 5, 3-10

Nguyen, M.P., Lee, S., and Lee, Y.M. (2013). Epigenetic regulation of hypoxia inducible factor in diseases and therapeutics. Arch. Pharm. Res. 36, 252-263.

Rundhaug, J.E. (2003). Matrix metalloproteinases, angiogenesis, and cancer. Clin. Cancer Res. 9, 551-554.

Schaller, M.D. (2001). Paxillin: a focal adhesion-associated adaptor protein. Oncogene 20,6459-6472.

Sigalotti, L., Fratta, E., Coral, S., and Maio, M. (2014). Epigenetic drugs as immunomodulators for combination therapies in solid tumors. Pharmacol. Ther. 142, 339-350.

Stroka, D.M., Burkhardt, T., Desbaillets, I., Wenger, R.H., Neil, D.A.H., Bauer, C., Gassmann, M., and Caninas, D. (2001). HIF1 is expressed in normoxic tissue and displays an organ-specific regulation under systemic hypoxia. FASEB J. 15, 2445-2453.

Tavora, B., Batista, S., Reynolds, L.E., Jadeja, S., Robinson, S., Kostourou, V., Hart, I., Fruttiger, M., Parsons, M., and HodivalaDilke, K.M. (2010). Endothelial FAK is required for tumour angiogenesis. EMBO Mol. Med. 2, 516-52840.
Tong, Z., Qing, Y., Wu, Y., Hu, X., Jiang, L., and Wu, X. (2014) Dioscin inhibits colon tumor growth and tumor angiogenesis through regulating VEGFR2 and AKT/MAPK signaling pathways. Toxicol. Appl. Pharmacol. 281, 166-173.

Varier, R.A., and Timmers, H.T.M. (2011). Histone lysine methylation and demethylation pathways in cancer. Biochim. Biophys. Acta $1815,75-89$

Wang, L., Zhang, Z.G., Zhang, R.L., Gregg, S.R., Hozeska-Solgot, A., Tourneau, Y.L., Wang, Y., and Chopp, M. (2006). Matrix metalloproteinase 2 (MMP2) and MMP9 secreted by erythropoietin-activated endothelial cells promote neural progenitor cell migration. N. Neurosci. 26, 5996-6003.

Watson, J.A., Watson, C.J., McCann, A., and Baugh, J. (2010). Epigenetics, the epicenter of the hypoxic response. Epigenetics 5, 293-296.

Xu, W.S., Parmigiani, R.B., and Marks, P.A. (2007). Histone deacetylase inhibitors: molecular mechanisms of action. Oncogene 26, 5541-5552.

Yee, K.M., Spivak-Kroizman, T.R. and Powis, G. (2008). HIF-1 regulation: not so easy come, easy go. Trends Biochem. Sci. 33, 526-534. 